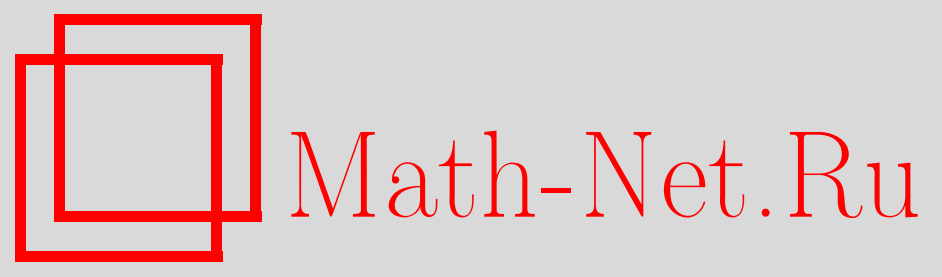

А. С. Соболев, Доказательство гипотезы Дирака для одного класса конечномерных систем Гамильтона-Дирака, $M$ тем. заметки, 2002, том 71, выпуск 5, 793-797

DOI: https://doi.org/10.4213/mzm654

Использование Общероссийского математического портала Math-Net.Ru подразумевает, что вы прочитали и согласны с пользовательским соглашением http://www.mathnet.ru/rus/agreement

Параметры загрузки:

IP : 3.91 .87 .62

26 апреля 2023 г., 12:59:44

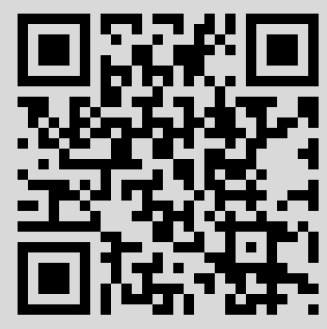




\section{ДОКАЗАТЕЛЬСТВО ГИПОТЕЗЫ ДИРАКА ДЛЯ ОДНОГО КЛАССА КОНЕЧНОМЕРНЫХ СИСТЕМ ГАМИЛЬТОНА-ДИРАКА}

\section{А. С. Соболев}

Введение. В статье доказано утверждение, впервые сформулированное Дираком в виде гипотезы в работе [1], в которой он изучал гамильтоновы системы со связями; теперь эти системы известны как системы Гамильтона-Дирака (СГД) (ср. [2] и [3]). В основе доказательства лежит метод, использующий теорию управляемых систем. Идея использования этого метода была заимствована из работы [4], однако в предлагаемом доказательстве применяется новый подход, который позволил доказать требуемое утверждение для более шарокого класса СГД (мы называем его классом Дирака), чем это сделано в [4].

1. Основные определения и обозначения. Пусть $E$ и $E_{\Phi}$ - евклидовы пространства.

ОПРЕДЕЛЕНИЕ 1. Связи в окрестности точки $x_{0} \in E$-это отображение $\Phi: E \rightarrow E_{\Phi}$, удовлетворяющее следуюшим условиям:

$\Phi$ непрерывно диффференцируемо в окрестности точки $x_{0}$ и $\Phi\left(x_{0}\right)=0$;

отображение $\Phi^{\prime}\left(x_{0}\right): E \rightarrow E_{\Phi}$ сюръективно.

ОПРЕДЕЛЕНИЕ 2. Система Гамильтона-Дирака на фазовом пространстве $E$ - это набор $(H, \Phi, E, I)$, где $H: E \rightarrow \mathbb{R}^{1}$ - гладкая функция, назьваемая гамильтонианом, $\Phi: E \rightarrow E_{\Phi}-$ гладкое отображение, удовлетворяющее определению связей, $E$ - четномерное евклидово пространство, $I: E \rightarrow E$ - симплектический оператор (ср. [2] и [3]).

Множество $\Gamma_{\Phi}=\{x \in E: \Phi(x)=0\}$ называется поверхностью связей. Определим функцию $\mathscr{H}: E \times E_{\Phi} \rightarrow \mathbb{R}^{1}$ равенством $\mathscr{H}(x, \lambda)=H(x)+\Phi(x) \lambda, \lambda \in E_{\Phi}$. Будем говорить, что функция $x(\cdot): \mathbb{R}^{1} \rightarrow E$ задает закон изменения состояния механической системы, описываемой СГД $(H, \Phi, E, I)$, если найдется функция $\lambda(\cdot): \mathbb{R}^{1} \rightarrow E_{\Phi}$ такая, что пара функций $(x(\cdot), \lambda(\cdot))$ удовлетворяет системе уравнений Гамильтона-Дирака (СУГД):

$$
\dot{x}(t)=I \cdot\left(\mathscr{H}_{E}^{\prime}\right)^{*}(x(t), \lambda(t)), \quad \Phi(x(t))=0 .
$$

Функция $\mathscr{H}$ называется обобщенным гамильтонианом. Для $\mathscr{H}$ будем полагать по определению, что $\{F, \mathscr{H}\}(x, \lambda)=\{F, H\}(x)+\{F, \Phi\}(x)(\lambda)$, где $F$ - гладкая функция на $E,\{\cdot, \cdot\}(x)-$ обычная скобка Пуассона, задаваемая симплектическим оператором. Зафиксируем фазовое пространство $E=Q \times P, Q=P=\mathbb{R}^{N}$, и оператор $I: I(p, q)=(q,-p)$.

2. Системы Гамильтона-Дирака. В общем случае не через каждую точку $\Gamma_{\Phi}$ проходит решение СУГД для СГД $(H, \Phi)$, и не для любой функции $\lambda(\cdot)$ существует решение СУГД. Задача определения полной поверхности связей (через каждую точку которой проходит решение) и класса допустимых функций $\lambda(\cdot)$ решается с помошью так называемой процедуры выявления связей (ПВС), в основе которой лежат уравнения сохранения связей во времени. Мы придерживаемся схемы ПВС, предложенной Дираком (см., также, [4]).

(C) А.С. Соволев

2002 
Пусть на $s$-м шаге ПВС получены новые связи $\Psi^{s}$, обобщенный гамильтониан $\mathscr{H}^{s}$ и поверхность связей $\Gamma_{\Psi^{0, \ldots, s}}$; здесь $\Psi^{0}=\Phi$. При выполнении некоторых условий уравнение сохранения связей $\Psi^{s}$ во времени $\left\{\Psi^{s}, \mathscr{H}^{s}\right\}\left(q, p, \lambda^{s}\right)=0$ равносильно равенствам $\lambda_{1}^{s}=\hat{\lambda}_{1}^{s}\left(q, p, \lambda_{2}^{s}\right)$ и $\widetilde{\Psi}^{s+1}(q, p)=0$, где $\lambda^{s}=\left(\lambda_{1}^{s}, \lambda_{2}^{s}\right)$. Будем предполагать, что в окрестности некоторой точки множество $\Gamma_{\Psi^{0, \ldots, s}} \cap \Gamma_{\widetilde{\Psi} s+1}$ является гладкой поверхностью; в этом случае найдется отображение $\Psi^{s+1}$ такое, что отображение $\Psi^{0, \ldots, s+1}:=\left(\Psi^{0, \ldots, s}, \Psi^{s+1}\right)$ удовлетворяет определению связей, и в этой окрестности $\Gamma_{\Psi^{0, \ldots, s+1}}=\Gamma_{\Psi^{0, \ldots, s}} \cap \Gamma_{\widetilde{\Psi} s+1}$.

Будем говорить, что СГД $(H, \Phi)$ выдерживает ПВС, если на каждом $(s+1)$-м шаге ПВС функции $\mathscr{H}^{s}, \Psi^{s}$ и $\widetilde{\Psi}^{s+1}$ удовлетворяют всем сформулированным выше условиям. Обозначим через $\Psi$ все новые связи, они называются вторичным.ми; при этом связи $\Phi$ назьваются первичныцми. Все связи $(\Phi, \Psi)$ будем также обозначать через $\Phi^{*}$. Функцию на фазовом пространстве, коммутирующую со всеми связями на поверхности связей $\Gamma_{\Phi^{*}}$, будем называть величиной первого рода, любую систему функций $\chi=\left(\chi_{1}, \ldots, \chi_{k}\right)$, для которой матрица $\|\{\chi, \chi\}\|$ является неособенной на $\Gamma_{\Phi^{*}}$, будем называть системой $у$ нкиий второго рода. Дирак показал, что связи $\Phi$ и $\Psi$ можно разделить на связи первого и второго рода, мы будем обозначать их через $\left(\Phi_{\mathrm{I}}, \Phi_{\mathrm{II}}\right)$ и $\left(\Psi_{\mathrm{I}}, \Psi_{\mathrm{II}}\right)$.

Связи $\Psi^{s}$, полученные на $s$-м шаге, в окрестности точки $\left(q_{0}, p_{0}\right) \in \Gamma_{\Phi *}$ также можно разделить на связи первого и второго рода, т.е. представить их в виде $\left(\Psi_{\mathrm{I}}^{s}, \Psi_{\mathrm{II}}^{s}\right)$. Применяя к $\Psi^{s}$ шаг процедуры выявления связей, получим новые связи $\left(\Psi_{\mathrm{I}}^{s+1}, \Psi_{\mathrm{II}}^{s+1}\right)$. Рассмотрим, кроме того, отдельно уравнения сохранения связей $\Psi_{\mathrm{I}}^{s}$ во времени. Пусть непосредственным следствием этих уравнений являются равенства $\widetilde{\Psi}^{s+1, \mathrm{I}}(q, p)=0$. Мы будем говорить, что связи первого рода, полученные на $(s+1)$-м шаге, невырождены и не являются следствиями сохранения во времени связей второго рода, если в качестве функций $\Psi_{\mathrm{I}}^{s+1}$ можно взять некоторые из функций $\widetilde{\Psi}^{s+1, \mathrm{I}}$.

ОПРЕДЕЛЕНИЕ 3. СГД $(H, \Phi)$ принадлежит жлассу Дирака, если она удовлетворяет следующим условиям:

функции $H$ и $\Phi$ аналитические;

СГД $(H, \Phi)$ выдерживает процедуру выявления связей;

никакая связь первого рода не является следствием сохранения во времени связи второго рода, и все связи первого рода, получаемые на каждом шаге процедуры выявления связей, невырождены.

\section{3. Гипотеза Дирака.}

ОПРЕДЕЛЕНИЕ 4. Аналитическая функция $f^{p h}$, зависящая от $q, p, \ldots, q^{(k)}, p^{(k)}$, назьвается физической величиной для СГД $(H, \Phi)$, если для любых двух гладких пересекающихся решений $(q(\cdot), p(\cdot))$ и $(\widetilde{q}(\cdot), \widetilde{p}(\cdot))$ СУГД выполняется тождество

$$
f^{p h}\left(q(t), p(t), \ldots, q^{(k)}(t), p^{(k)}(t)\right) \equiv f^{p h}\left(\tilde{q}(t), \tilde{p}(t), \ldots, \tilde{q}^{(k)}(t), \tilde{p}^{(k)}(t)\right) .
$$

Пусть траектория $(\tilde{q}(\cdot, \varepsilon), \tilde{p}(\cdot, \varepsilon))$ получена из $(q(\cdot), p(\cdot))$ с помощью канонического преобразования с производящей функцией

$$
F(q, \tilde{p}, t, \varepsilon)=\sum_{i=1}^{N} q_{i} \tilde{p}_{i}+\varepsilon W(q, \tilde{p}, t),
$$

где $W(q, \tilde{p}, t)=a_{k}(q, \tilde{p}) \gamma^{k}(t)$. Пусть $g$-произвольная гладкая функция на фазовом пространстве. Будем говорить, что функция $g$ не меняет своего значения при бесконечно малых канонических преобразованиях траектории $(q(\cdot), p(\cdot))$, генерируемых $a_{k}$, если

$$
\left.\frac{d}{d \varepsilon}(g(\tilde{q}(t, \varepsilon), \tilde{p}(t, \varepsilon))-g(q(t), p(t)))\right|_{\varepsilon=0}=0 .
$$

Теорема 1 (гипотеза Дирака). Пусть СГД $(H, \Phi)$ принадлежит классу Дирака. Тогда

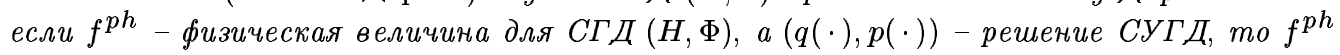
не меняет своего значения при бесконечно малых канонических преобразованиях траектории $(q(\cdot), p(\cdot))$, генерируемых всеми связями первого рода. 
Лемма 1. Имеет место равенство

$$
\left.\frac{d}{d \varepsilon}(g(\tilde{q}(t, \varepsilon), \tilde{p}(t, \varepsilon))-g(q(t), p(t)))\right|_{\varepsilon=0}=\left\{g, a_{k}\right\}(q(t), p(t)) \gamma^{k}(t) .
$$

4. Канонические переменные. Пусть СГД $(H, \Phi)$ принадлежит классу Дирака. Без ограничения общности можно считать, что $(0,0) \in \Gamma_{\Phi^{*}}$, все дальнейшие рассуждения справедливы в некоторой окрестности точки $(0,0)$. Мы воспользуемся каноническими переменными $(\omega, Q, \Omega)$, введенными в $[4, \S 5]$. Они получаются с помощью канонического преобразования, выбранного таким образом, чтобы в новых координатах связи $\Phi^{*}$ были эквивалентны $\Omega$, а связи $\Phi$ - эквивалентны $\Omega^{(1)}$. В новых координатах $\Omega^{(1)}$ и $\Omega^{(2)}$ - первичные и вторичные связи, $P^{(1)}$ и $P^{(2)}$ - первичные и вторичные связи первого рода, $Q$ сопряжены $P$. Мы считаем, что вторичные связи первого рода существуют, иначе утверждение гипотезы Дирака тривиально. Рассуждения из [4, гл. 2, § 5] показывают, что в новых координатах обобщенный гамильтониан имеет вид

$$
\mathscr{H}(\omega, Q, \Omega, \lambda)=H^{p h}(\omega)+P^{(2)} A(\omega, Q)+\Delta \mathscr{H}+\left(\lambda_{\Omega^{(1)}}, \Omega^{(1)}\right)
$$

(мы будем обозначать его так же, как и обобщенный гамильтониан в исходных переменных); здесь $\Delta \mathscr{H}$ - часть $\mathscr{H}$, не менее чем квадратичная по связям $\Omega^{(2)}$ и не зависящая от связей $\Omega^{(1)}$.

Исследуем процесс выявления связей в СГД. Имеем

$$
\widetilde{\Psi}^{1, \mathrm{I}}(\omega, Q, \Omega)=-P^{(2)} \mathrm{A}_{1}(\omega, Q)-F_{1}(\omega, Q, \Omega)
$$

здесь $F_{1}$ не менее чем квадратична по $\Omega^{(2)}$. Так как СГД принадлежит классу Дирака, то все новые связи первого рода $\Psi_{\mathrm{I}}^{1}$ могут быть выбраны из функций $\widetilde{\Psi}^{1, \mathrm{I}}$. Можно показать, что уравнения $\left\{\widetilde{\Psi}^{1, \mathrm{I}}, \mathscr{H}^{1}\right\}(\omega, Q, \Omega)=0$ и $\left\{\Psi_{\mathrm{I}}^{1}, \mathscr{H}^{1}\right\}(\omega, Q, \Omega)=0$ на множестве $\Gamma_{\Phi, \Psi^{1}}$ эквивалентны, поэтому для удобства мы будем использовать уравнение сохранения $\widetilde{\Psi}^{1, \mathrm{I}}$ во времени. Функции $\widetilde{\Psi}^{2, \mathrm{I}}$, являющиеся следствиями этого уравнения, имеют аналогичный вид

$$
\widetilde{\Psi}^{1, \mathrm{I}}: \widetilde{\Psi}^{2, \mathrm{I}}(\omega, Q, \Omega)=-P^{(2)} \mathrm{A}_{2}(\omega, Q)-F_{2}(\omega, Q, \Omega),
$$

и к ним применимы аналогичные утверждения. В резултате процедуры выявления связей мы получим наборы функций $\widetilde{\Psi}^{k, \mathrm{I}}$ и $\Psi_{\mathrm{II}}^{k}, k=1, \ldots, s_{0}$. Равенства $\widetilde{\Psi}^{k, \mathrm{I}}(\omega, Q, \Omega)=0, k=1, \ldots, s_{0}$, равносильны равенству $P^{(2)} \bar{A}(\omega, Q)+\bar{F}(\omega, Q, \Omega)=0$, где $\bar{A}(\omega, Q)=\left(A_{1}(\omega, Q), \ldots, A_{s_{0}}(\omega, Q)\right)$, $\bar{F}(\omega, Q, \Omega)=\left(F_{1}(\omega, Q, \Omega), \ldots, F_{s_{0}}(\omega, Q, \Omega)\right)$. Так как вторичные связи первого рода нашей СГД эквивалентны $P^{(2)}$, то в окрестности нуля ранг матрицы $\bar{A}$ равен $\#\left[P^{(2)}\right]$ (количество переменных $\left.P^{(2)}\right)$.

\section{5. Вполне управляемые системы.}

ОПРЕДЕЛЕНИЕ 5 . Процесс $\dot{x}=A(t, x)+B u(t)$, где В: $\mathbb{R}^{M} \rightarrow \mathbb{R}^{N}$ - постоянная матрица, назьвается локально вполне управляемым вдоль решения $x(\cdot)$, соответствуюшего управлению $u_{0}(\cdot)$, на отрезке $\left[t_{0}, t_{1}\right]$, если для любого $\varepsilon>0$ найдется $\delta>0$ такое, что каждая точка $x$, для которой $\left|x-x\left(t_{1}\right)\right|<\delta$, достигается из точки $x_{0}=x\left(t_{0}\right)$ под действием гладкого управления $u(\cdot)$, удовлетворяющего условию $\left|u(t)-u_{0}(t)\right|<\varepsilon$ на отрезке $t_{0} \leqslant t \leqslant t_{1}$.

ЛЕмма 2. Прочесс $\dot{x}=A(t, x)+B u(t)$ является локально вполне управляемым вдоль решения $x_{0}(\cdot)$, соответствующего управлению $u(\cdot)$, на отрезке $\left[t_{0}, t_{1}\right]$, если не существует постоянного вектора $w \in \mathbb{R}^{N}$ такого, что $w \neq 0 u \mathrm{wI}^{-1}(t) B \equiv 0$ на отрезке $\left[t_{0}, t_{1}\right]$, где $I(\cdot)$ - фундаментальное решение уравнений

$$
\dot{y}=\frac{\partial A}{\partial x}\left(t, x_{0}(t)\right) y
$$

с начальным условием $I\left(t_{0}\right)=\mathrm{id}$. 
ДоКАЗАТЕльство. Надо воспользоваться идеей доказательства теоремы 1 в [5, глава $6, \S 1]$, а затем теоремой 6 в [5, глава $3, \S 2]$.

6. Доказательство гипотезы Дирака. Пусть СГД $(H, \Phi)$ принадлежит классу Дирака. Преобразуем ее к каноническим координатам и проведем процедуру выявления связей. В результате СУГД преобразуется в эквивалентную ей систему:

$$
\dot{\omega}=\left\{\omega, H^{p h}\right\}, \quad \dot{Q}^{(1)}=\lambda_{P^{(1)}}, \quad \dot{Q}^{(2)}=A(\omega, Q), \quad \Omega=0 .
$$

Определим $n_{1}=\#\left[P^{(1)}\right]$ и $n_{2}=\#\left[P^{(2)}\right]$. Запишем уравнения для $Q^{(1)}$ и $Q^{(2)}$ в виде

$$
\dot{Q}=\widetilde{A}(\omega, Q)+B \lambda_{P^{(1)}}(t),
$$

где $\widetilde{A}=(0, A) \in \mathbb{R}^{n_{1}+n_{2}}, B: \mathbb{R}^{n_{1}} \rightarrow \mathbb{R}^{n_{1}+n_{2}}$ определяется равенством $B x=(x, 0)$. Это уравнение будем назьвать $Q$-процессом. Пусть $\omega(\cdot)$-произвольное решение системы $\dot{\omega}=\left\{\omega, H^{p h}\right\}$ на отрезке $\left[t_{0}, t_{1}\right], Q(\cdot)$ - решение системы $\dot{Q}=\widetilde{A}(\omega(t), Q)$ на $\left[t_{0}, t_{1}\right]$.

ЛЕмма 3. Пусть СГД $(H, \Phi)$ принадлежит классу Дирака и преобразована к каноническим переменным. Пусть $I(\cdot)$ - фундаментальное решение системь

$$
\dot{x}(t)=\frac{\partial \widetilde{A}}{\partial Q}(\omega(t), Q(t)) x(t)
$$

на отрезке $\left[t_{0}, t_{1}\right]$, удовлетворяющее начальному условию $I\left(t_{0}\right)=\mathrm{id}$. Тогда не существует вектора $w \in \mathbb{R}^{n_{1}+n_{2}}, w \neq 0$, такого, что $w I^{-1}(t) B \equiv 0$ на $\left[t_{0}, t_{1}\right]$.

ДоКАЗАТЕЛЬСтво. Предположим, что такой вектор $w$ существует, и обозначим через $w^{(1)}$ первые $n_{1}$ его компонент, а через $w^{(2)}$ последние $n_{2}$ его компонент. Из тождества $w I^{-1}(t) B \equiv 0$ следует, что все производные выражения $w I^{-1}(t) B$ обращаются в нуль в точке $t_{0}$. Очевидно,

$$
\left.w I^{-1}(t) B\right|_{t=t_{0}}=w^{(1)}=0,\left.\quad\left(w I^{-1}(t) B\right)^{\prime}\right|_{t=t_{0}}=-w^{(2)} \frac{\partial A}{\partial Q^{(1)}}\left(\omega\left(t_{0}\right), Q\left(t_{0}\right)\right)=0 .
$$

Непосредственно проверяется, что

$$
\left.\left(w I^{-1}(t) B\right)^{(k+1)}\right|_{t=t_{0}}=-w^{(2)} E_{k+1}\left(t_{0}\right),
$$

где

Напомним, что

$$
E_{k+1}(t)=\dot{E}_{k}(t)-\frac{\partial A}{\partial Q^{(2)}}(\omega(t), Q(t)) E_{k}(t), \quad E_{1}(t)=\frac{\partial A}{\partial Q^{(1)}}(\omega(t), Q(t)) .
$$

$$
\widetilde{\Psi}^{1, \mathrm{I}}=-P^{(2)} \frac{\partial A}{\partial Q^{(1)}}-F_{1}, \quad \widetilde{\Psi}^{2, \mathrm{I}}=-\widetilde{F}_{2}-P^{(2)}\left\{\frac{\partial A}{\partial Q^{(1)}}, H^{p h}+P^{(2)} A\right\}+P^{(2)} \frac{\partial A}{\partial Q^{(2)}} \frac{\partial A}{\partial Q^{(1)}} .
$$

Для любой функции $G$ переменных $(\omega, Q)$ (в том числе матричнозначной) справедливо равенство

$$
\left\{G, H^{p h}+P^{(2)} A\right\}\left(\omega\left(t_{0}\right), Q\left(t_{0}\right), P_{0}^{(2)}\right)=\dot{G}\left(\omega\left(t_{0}\right), Q\left(t_{0}\right)\right)+\sum_{i}\left\{G, A_{i}\right\}\left(\omega\left(t_{0}\right), Q\left(t_{0}\right)\right)\left(P_{0}^{(2)}\right)_{i}
$$

Тогда

$$
\widetilde{\Psi}^{2, \mathrm{I}}\left(\omega\left(t_{0}\right), Q\left(t_{0}\right), \Omega\right)=-P^{(2)} E_{2}\left(t_{0}\right)-F_{2}\left(\omega\left(t_{0}\right), Q\left(t_{0}\right), \Omega\right),
$$

т.е. равенство $\widetilde{\Psi}^{2, \mathrm{I}}\left(\omega\left(t_{0}\right), Q\left(t_{0}\right), \Omega\right)=0$ с точностью до квадратичной по $\Omega^{(2)}$ части совпадает с равенством $\left.\left(w I^{-1}(t) B\right)^{(2)}\right|_{t=t_{0}}=0$. Можно доказать, что то же самое верно для всех $\widetilde{\Psi}^{k, \mathrm{I}}$ и $\left(w I^{-1} B\right)^{(k)}$, т.е. $E_{k}\left(t_{0}\right)=A_{k}\left(\omega\left(t_{0}\right), Q\left(t_{0}\right)\right)$ для всех $k$. Было показано, что ранг матрицы $\bar{A}$ в каждой точке из окрестности нуля равен $\#\left[P^{(2)}\right]=\#\left[w^{(2)}\right]$. Следовательно, ранг аналогич-

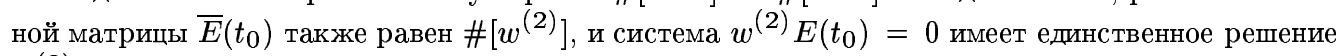
$w^{(2)}=0$, что противоречит предположению.

Из лемм 2 и 3 следует, что $Q$-процесс является локально вполне управляемым вдоль решения $Q(\cdot)$ с управляющей функцией $\lambda_{P^{(1)}}(\cdot) \equiv 0$. Пусть $\tilde{f}^{p h}$ - физическая величина для СГД $(H, \Phi)$; нетрудно показать (см. $[4, \S 7])$, что на решениях СУГД $\tilde{f}^{p h}$ совпадает с некоторой физической величиной $f^{p h}$, зависящей только от $\omega, Q^{(1)}$ и производных $Q^{(1)}$. 
ЛЕмма 4. Физическая величина $f^{\text {ph }}$ зависит только от $\omega$.

ДокАЗАТЕЛЬство. Пусть $\left(\omega_{1}, Q_{1}\right)$ - произвольная точка на поверхности связей, $\omega(\cdot)-$ решение системы $\dot{\omega}=\left\{\omega, H^{p h}\right\}$ с начальным условием $\omega\left(t_{1}\right)=\omega_{1}, Q(\cdot)$-решение системы $\dot{Q}=$ $\widetilde{A}(\omega(t), Q)$ с начальным условием $Q\left(t_{1}\right)=Q_{1}$. Возьмем $t_{0}: t_{0}<t_{1}$ и обозначим $\omega_{0}=\omega\left(t_{0}\right)$, $Q_{0}=Q\left(t_{0}\right)$. Определим

$$
\pi: \lambda_{P^{(1)}}(\cdot) \mapsto Q_{Q_{0}, \lambda_{P}^{(1)}}\left(t_{1}\right), \quad \tau: \lambda_{P^{(1)}} \mapsto\left(\pi\left(\lambda_{P^{(1)}}\right), \lambda_{P^{(1)}}^{\prime}\left(t_{1}\right), \ldots, \lambda_{P^{(1)}}^{(k)}\left(t_{1}\right)\right) .
$$

Так как $Q$-процесс локально вполне управляем, то образ $\pi$ содержит окрестность $O$ точки $Q_{1}$; отсюда и из непрерьвности $\pi$ следует, что образ $\tau$ всюду плотен в $O\left(Q_{1}\right) \times \mathbb{R}^{k n_{1}}$. Траектории $(\omega(t), Q(t))$ и $\left(\omega(t), Q_{Q_{0}, \lambda_{P}(1)}(t)\right)$ пересекаются в точке $\left(\omega_{0}, Q_{0}\right)$; используя определение физической величины, при $t=t_{1}$ получим равенство $f\left(\omega_{1}, Q_{1}, 0, \ldots, 0\right)=f\left(\omega_{1}, Q_{1}+\Delta, \delta^{1}, \ldots, \delta^{k}\right)$, которое вьполняется для всюду плотного в $O(0) \times \mathbb{R}^{k n_{1}}$ множества $\left(\Delta, \delta^{1}, \ldots, \delta^{k}\right)$. Из непрерьвности $f$ следует ее независимость от $Q^{(1)}$ и от производных $Q^{(1)}$.

СледствиЕ. На решениях СУГД любая физическая величина совпадает с некоторой функцией, определенной только на фазовом пространстве.

ЛЕмма 5. Если $F^{p h}: Q \times P \rightarrow \mathbb{R}^{1}$ - физическая величина для СГД $(H, \Phi)$, принадлежсащ, ей классу Дирака, mo $\left.\left\{F^{p h},\left(\Phi_{\mathrm{I}}^{*}\right)_{i}\right\}\right|_{\Gamma_{\Phi^{*}}} \equiv 0$.

ДокАЗАТЕЛЬство. В канонических переменных

$$
\begin{gathered}
F^{p h}(q, p)=f_{F}^{p h}(\omega(q, p))+\Omega g_{F}(\omega(q, p), Q(q, p), \Omega(q, p)) \\
\left(\Phi_{\mathrm{I}}^{*}\right)_{i}(q, p)=\hat{\phi}_{i}(\omega(q, p), Q(q, p), \Omega(q, p)),
\end{gathered}
$$

где $\hat{\phi}_{i}$ - линейная комбинация связей первого рода. Тогда

$$
\left.\left\{F^{p h},\left(\Phi_{\mathrm{I}}^{*}\right)_{i}\right\}\right|_{\Gamma_{\Phi^{*}}}=\left.\left\{f_{F}^{p h}(\omega)+\Omega g_{F}(\omega, Q, \Omega), \hat{\phi}_{i}(\omega, Q, \Omega)\right\}\right|_{\Omega=0}=0 .
$$

Из лемм 1 и 5 следует справедливость гипотезы Дирака для СГД из класса Дирака.

\section{СПИСОК ЦИТИРОВАННОЙ ЛИТЕРАТУРЫ}

1. Dirac P. A. M. Lectures on Quantum Mechanics. New York: Belfer Graduate School of Science, 1964. 2. Альбеверио С., Смолянов О. Г. // Докл. РАН. 1997. Т. 355. № 1. С. 7-11. 3. Альбеверио С., Смолянов О. Г., Хренников А. Ю. // Докл. РАН. 2001. Т. 381 . № 2. С. 1-5. 4. Гитман Д. М., Тютин И. В. Каноническое квантование полей со связями. М.: Наука, 1986. 5. Ли Э. Б., Маркус Л. Основы теории оптимального управления. М.: Наука, 1972.

Московский государственный университет им. М. В. Ломоносова 